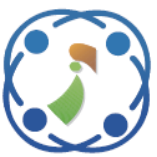

\title{
ANC System Modeling and Simulations in a Wide-Area Virtual Environment Using FDTD Approach with Perfectly Matched Layer Absorbing Boundaries
}

\author{
Chadaporn Sookpuwong ${ }^{1}$ \\ Chow Chompoo-inwai ${ }^{1 *}$ \\ ${ }^{1}$ Electrical Engineering Department, School of Engineering, \\ King Mongkut's Institute of Technology Ladkrabang (KMITL), Thailand \\ * Corresponding author's Email: chow.ch@kmitl.ac.th
}

\begin{abstract}
This paper presents a model and simulation results of the active noise control (ANC) system in a wide-area virtual environment using the acoustics finite-difference time-domain (FDTD) propagation approach defined by particle velocities and sound pressure level (SPL) within a certain absorbing boundary conditions called a perfectlymatched layer (PML) technique. The ANC excitation applies a single-frequency noise source with an adaptive feedforward configuration. The FDTD algorithm is used to model the area of interest acoustically, including a desired quiet zone, considering the effects from a primary path, a secondary path, and a feedback path. A processing unit of the ANC system based on the least mean-squared (LMS) algorithm is utilized to synthesize a cancelling noise using a secondary loudspeaker. A single-channel feedforward ANC system used in this paper is modified from the proposed multi-channel models and forms. Acoustics FDTD propagation results can be used to determine the optimum placements for ANC sensors and actuators. The SPL numerical and graphical results are plotted to demonstrate the performance of the proposed ANC system. All of the simulation results confirm that the proposed modeling approach can be combined with wide-area acoustic simulations and a feedforward LMS adaptive algorithm. The proposed model also provides a way for the optimum placement of the ANC sensors and actuators before being used in a more-complex practical environment.
\end{abstract}

Keywords: Active noise control (ANC), Finite-difference time-domain (FDTD), Single-channel feedforward ANC system, Perfectly-matched layer (PML), Absorbing boundary, Sound pressure level (SPL).

\section{Introduction}

Nowadays, active noise control (ANC) technique has become more and more popular and widely been used to diminish or cancel unwanted acoustic noises. In early stage, a German engineer and entrepreneur, Pual Lueg, patented process on silencing oscillation sounds using a superposition concept. He introduced the additional sound, called secondary sources, which equal in magnitude but opposite in phase, to cancel out the unwanted noises in 1936 [1]. At the time, his technique had many difficulties to implement due to the complication of analog-and-electronics devices which were used to create the secondary sources. Until 1975, Bernard Widrow, a professor at Stanford university, proposed an inventive least mean square (LMS) adaptive algorithm that is a part of an adaptive filter and also published the alternative noise cancelling algorithm using his adaptive technique to estimate signal corrupted by additive interference noises. Widrow's technique was then be counted as the first of many ANC systems in the digital signal processing (DSP) world [2]. Classical ANC concepts from Pual Lueg and Bernard Widrow will be shown in Fig. 1. Recently, the ANC has become one of the most favourite tools to resolve noise problems in many engineering applications for example: noisecancellation headphones, noise control systems in cars, airplanes and aircraft cockpits, a variety of soundproof equipment, and etc. A general ANC architecture comprises of one or many reference sensors, cancelling loudspeakers, error sensors, and controllers. Today, two types of ANC architectures have widely been used namely feedforward and feedback ANC systems [3]. Either of a single-channel 
or a multi-channel ANC system will be chosen depending on the level of problem complications. In this paper, a single-channel feedforward ANC system will be utilized, more details in Section 2 .

During the past three decades, the ANC relevant research topics have been broadened and enlarged in many ways. Some researchers mainly focused on ANC algorithms improvements, for example, DahChung Chang presented the new FXLMS algorithm with a variable tap length and a step size to improve convergence rate and noise reduction ratio [4], Ardekani and Abdulla introduced the ANC with the emphasis on ANC system implemented using FXLMS adaptation algorithm [5]. Some researchers concentrated on expanding ANC applications, for example, Sen Kou applied a feedback active noise control technique for headphone applications [6], László Sujbert presented the ANC noise-cancelling office chair to resolve the uncomfortable problem of employees wearing a noise-cancelling headphone in the office using a multiple reference microphones technique [7], Chraponska, Wrona, Rzepecki, Mazur and Pawelczyk proposed the active structural acoustic control of ANC active casing placed in the corner to protect the low-frequency noise from electrical appliances [8].

The term wide area for ANC application was first introduced by Graupe and Efron in 1991 [9], they proposed the method of using an output-whitening approach ANC system in the vicinity of noisy machinery. Three years later, Efron and Han proposed the extension from his own work with Graupe on a single point adaptive ANC to address the problem of wide-area noise cancellation [10]. Currently, many research relevant to a wide-area-like ANC applications have been published in various aspects, for example, Gunnar Gäbel proposed the development and implementation of a multi-channel ANC system for the reduction of road induced vehicle interior noise [11], Liu and Kuo proposed the use of microphone array in wide area simulations [12], Iwai, Hase and Kajikawa tried to identify the optimal reference microphone array based on time difference arrival (TDOA) technique [13], Jianjun, Bhan, Dongyuan and Woon presented the leverage of an underdetermined system in a multi-channel ANC for open windows in a large space [14], Ying, Wang and Liu presented the application study of an adaptive tracking algorithm in the ANC system for lower the transformer noise in the substation specific area [15], Zhang, Qin, Zou and Qiu presented the secondary source consideration and error sensing strategies for the ANC of sound transmission through a small opening in a wall [16], Munir and Abdulla proposed new ANC algorithms to deal with acoustic domain in silencing a remote location quiet zone called FxLMS algorithm in 2020 [17]. Some researchers attempted to create a quiet zone in specific area like closed room using sparse decomposition of reference signals from distributed sensors arranged in term of circular arrays to reduce broadband noise fields [18-20]. Some researchers applied electromagnetic wave equations to explore the ANC propagation filed, for example, Szarvas and Sujbert [21] presented the efficiency testing of the ANC calculation by acoustic field modeling, Sankaran reviewed many computational tools on how to make a good choice for modelling electromagnetics wave propagation problem [22], Orlis, Giouvanakis and Papanikolaou presented the simulation of the ANC in a small and enclosed workplace and modeling using the Finite Difference Time Domain (FDTD) method [23]. It should be noted here that although many ANC research have been done and already published but almost all of the done research would mainly focus on purely acoustic domain performance using either the numerical or analytical methodologies and neglecting some important factors or parameters involving in the actual environment.

The main gold of this research is to utilize an original idea of a classical ANC concept to form a complete mathematical model and analysis in a wide area with remote location of a desired quiet zone. Studied from the previous research, some missing points or negligibility worth mentioning here. Firstly, for acoustic-domain analysis in a wide area, not only the primary path effect, but also the secondary path and the feedback path effects shall be considered as in reality the noise source and each of the ANC devices may not be in the same locations like other ANC applications. Secondly, to setup an accurate model for the acoustic propagating simulations, the artificial reflections in the space must be considered. Thirdly, to simultaneously observe true-propagation phenomena and the ANC system effectiveness, a real-time correlation between two domains should be integrated.

This research proposes together a combination analysis of a noise-propagations acoustic domain and the electrical device and ANC controller in time domain. In the acoustic-domain analysis, different from most of the aforementioned papers, we will include all of the primary path effect, the secondary path effect and the feedback path effect into our considerations. The discretized FDTD propagation equations will be derived and presented here. When applying the FDTD propagation to a wide-area virtual environment, there will be some artificial reflections occurred along the space borders. We then proposed the use of the absorbing boundary condition 
called a perfectly matched layer (PML) technique to resolve such a problem (more details on PML will be discussed in Sections 2 and 3). The integration of the FDTD propagation with the PML technique ensures the practical environment in our virtual simulation. A time-domain analysis includes the ANC algorithm and ANC devices i.e., a processor, a reference sensor, a cancelling loudspeaker, and an error sensor. A simple and effective least mean square (LMS) algorithm will be selected as an ANC algorithm to ensure the system stability [24-26].

The proposed two-domain combination model for wide-area analysis was done by discretizing the FDTD propagation equations combining with the PML technique then formulate another new set of equations for an ANC system with LMS algorithm, in time domain. Using this newly proposed model and technique, a variety of handy simulations and analysis can comfortably be achieved, for example, visualizations of sound pressure level fields, a noise attenuation (in $\mathrm{dB}$ ) and other relevant parameters at any point in the space including the remote area of interest (desired quiet zone). Another big advantage when using the proposed model and analysis is that locations of either a noise source or ANC devices can freely be placed in the space which will definitely be helpful to determine the optimum locations for ANC devices. The flexibility and capability by using the proposed model and analysis in a wide-area virtual environment offer a great number of possibilities for a future research to come.

This research paper organizations are as follows: first section is an introduction, Section 2 talks about the backgrounds and theories, Section 3 explains the proposed methodologies, Section 4 shows numerous and intensive simulation results and analysis, Section 5 mentions future work and discussions, and finally, Section 6 is a research summary and conclusions.

\section{Background and theories}

\subsection{Active noise control (ANC) principle}

Active noise control (ANC) is an active research area that focuses on removing contaminated sounds or vibrations in any environment with the use of sensors, actuators and advanced mathematical algorithms. Since 1936, Paul Lueg proposed and published a simple ANC system design to reduce the unwanted acoustic noise [1]. He proposed a technique for controlling unwanted sounds by introducing additional sounds. And when the advent of digital technologies did the realization, adaptive ANC

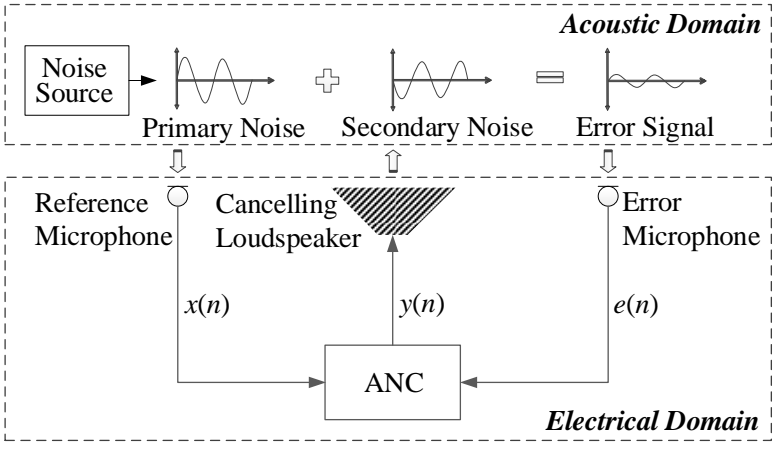

Figure. 1 A summary of a classical ANC system concept by Paul Lueg [1] and Widrow [11]

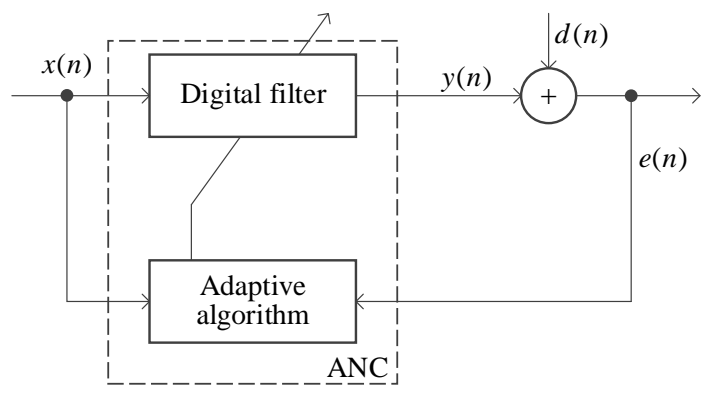

Figure. 2 A Simplified diagram of a classical ANC

systems which comprise of adaptive algorithm to adjust ANC device parameters become possible. Such an ANC technology was first published by Widrow in 1975 [2] as illustrated in Fig. 1. Since then, there had been many researchers proposed similar functions to Paul Lueg's ANC system with different configurations of reference microphones, different numbers and types of cancelling loudspeakers and different algorithms [3].

The most important compositions of the ANC architecture are the digital filter and adaptive algorithms which will be used to compute ANC system outputs. A general form of an ANC system is a time-varying system that applies a recursive (adaptive) algorithm to continuously adjust its coefficients (weights) for operation in an unknown environment over time.

\subsubsection{Digital filter}

Determine $d(n)$ is the desired signal, $y(n)$ is the estimated signal computed from the digital filter and adaptive algorithm, $x(n)$ is the reference signal or noise signal, and $e(n)$ is an error signal between $d(n)$ and $y(n)$, computed as shown in Eq. (1)

$$
e(n)=y(n)-d(n)
$$

From Fig. 2, the finite impulse response (FIR) filter will be used as the digital transversal filter. The FIR filtering block consists of a tapped-delay line and 
a set of tapped weights. The tapped-delay line gathers a block of $L$ samples which mathematically represented in a vector form shown in Eq. (2). Similarly, the tapped weights can be computed from Eq. (3).

$$
\begin{gathered}
\boldsymbol{x}(n)=[x(n), x(n-1), \ldots, x(n-L+1)]^{T} \\
\boldsymbol{w}(n)=\left[w_{0}(n), w_{1}(n), \ldots, w_{L-1}(n)\right]^{T}
\end{gathered}
$$

The parameter $L$ is the FIR filter length. Both $\mathbf{x}(n)$ and $\mathbf{w}(n)$ are defined as $L-b y-1$ column vector, where the index $n$ is the parenthesis represents the time index. The output $y(n)$ can be expressed in both scalar and vector notation as in Eq. (4).

$$
y(n)=\sum_{m=0}^{L-1} w_{m}(n) x(n-m)=\boldsymbol{w}^{T}(n) \boldsymbol{x}(n)
$$

\subsubsection{Adaptive algorithm}

In most of practical applications, filter specifications are unknown at the design step. A better solution for solving this problem is to use a digital filter with time-varying coefficients to track the unknown yet changing environments. Different from the fixed-coefficient filters, designing an adaptive filter requires some considerations i.e., the filter length, step size, regularization parameters and the adaptive algorithm. One of the most popular and widely use adaptive algorithms is the Least Mean Square (LMS) algorithm. The LMS algorithm updates the filter coefficients as in Eq. (5).

$$
\boldsymbol{w}(n+1)=\boldsymbol{w}(n)+\mu \boldsymbol{x}(n) e(n)
$$

Where $\mu$ is the step size that determines the stability and the convergence rate of the LMS algorithm. The time-varying nature of the weight vector $\mathbf{w}(n)$ is indicated by having an index $n$ to show its value as function of time. At the current iteration $n$, the amount of adjustment to the current tap weights $w(n)$ is determine by the input vector $\mathbf{x}(n)$ scaled with the estimated error $e(n)$ measures the deference between the adaptive filter output $y(n)$ and the desired response $d(n)$ [3].

\subsection{Multiple-channel feedforward ANC systems}

In general, there are two types of ANC systems popularly mentioned in many research and engineering solutions, i.e. feedforward and feedback ANC systems. In this article, we will mainly focus on a feedforward ANC system because of its good performances when using with broadband range of frequencies [3]. A feedforward ANC system is one

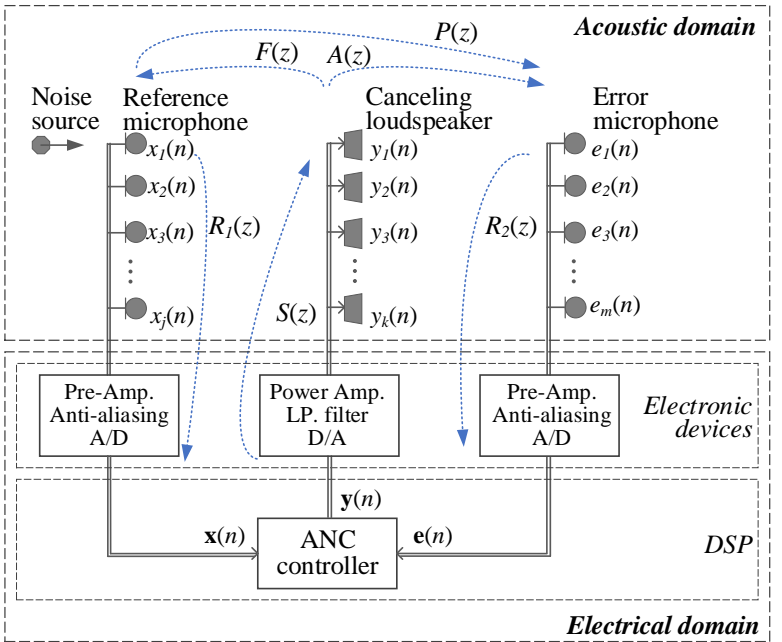

Figure. 3 An overview of a multiple-channel feedforward ANC system

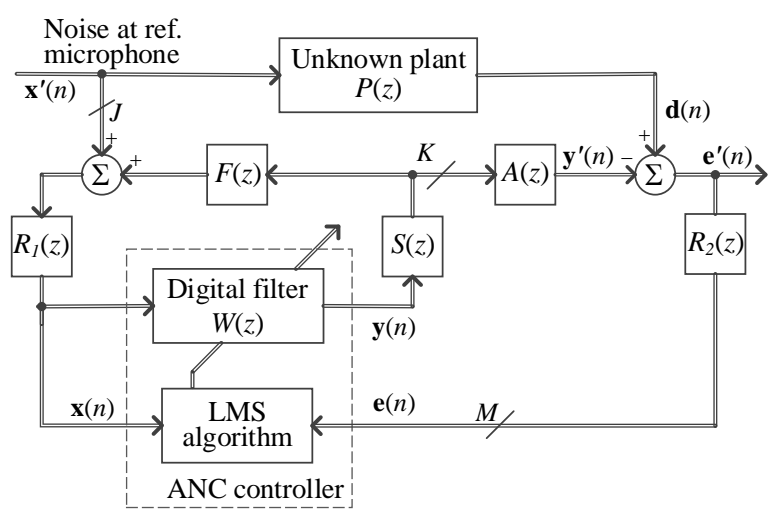

Figure. 4 A simplified diagram of a multiple-channel feedforward ANC system with LMS algorithm

kind of a modification from Paul Lueg's original ANC principle. Basic concepts and necessarily equations of a feedforward ANC system will be elaborated here using an architecture of a multiplechannel feedforward shown in Fig.3. although simulation results presented later in this paper will be the outcomes from a single-channel feedforward ANC system for simplicity reason. Typical compositions of a multiple-channel feedforward ANC system consists of several reference sensors, canceling loudspeakers, error sensors and one unit of ANC controller. Unwanted signals (noises) will be picked up through medium (air) by reference sensors and then passed to an ANC controller via electronics devices. Compensation signals will then be computed by a controller and sending out to drive a group of cancelling loudspeakers also via another set of electronics devices. Remaining acoustic noises after compensations will be picked up by error sensors and sent back to a controller again.

Fig. 3 illustrates an overview of a multi-channel feedforward ANC architecture arranged in wide areas. 
It can be seen from Fig. 3 that there are two important domains in this ANC architecture: electrical domain and acoustic domain. Many electronics devices including analog to digital (A/D) and digital to analog (D/A) circuits are presented here. The ANC controller with adaptive filters and algorithm is also counted as one part of this domain. The acoustic domain, on the other hand, more concerns will be based on acoustic propagations.

It should be stated here that all the acoustic propagation paths will directly affect the ANC performance. The first and most important acoustic part of interest for this feedforward ANC is called a primary path, $P(z)$, which is the propagating path from the noise sources or reference microphones to the error microphones. The secondary path, $A(z)$, in this system can be determined as the acoustic propagating path from cancelling loudspeakers to the error microphones. Since the anti-noise outputs from cancelling loudspeakers are multi-directional effects, the feedback propagating path, $F(z)$, from cancelling loudspeakers to the reference microphones must always be considered as well. Therefore, when this type of ANC system is in operations, multidirectional path effects from acoustics propagation in this space shall be investigated.

The ANC controller typically composes of adaptive filters and recursive algorithms. In this paper, the finite impulse response (FIR) filters which have only zeros, hence always stable, with a least mean square (LMS) algorithm will be used to perform the ANC function.

A multi-channel feedforward ANC architecture can be simplified as shown in Fig. 4. All the acoustics path effects will be represented in terms of primary path, $P(z)$, secondary path, $A(z)$ and feedback path, $F(z)$. All electronics path effects will be represented in the forms of $R_{l}(z), S(z)$ and $R_{2}(z)$. In this paper, all electronics paths will be counted as ideal paths (no losses) and can be neglected as mentioned in previous research [24-26]. The input signals $x_{j}(n)$ picked up by $J$ reference sensors can be expressed as Eqs. (6) and (7).

$$
\begin{gathered}
\boldsymbol{x}_{j}(n)=\left[x_{j}(n), x_{j}(n-1), \ldots, x_{j}(n-1+L)\right]^{T} \\
\boldsymbol{x}(n) \equiv\left[\boldsymbol{x}_{1}^{T}(n), \boldsymbol{x}_{2}^{T}(n), \ldots, \boldsymbol{x}_{J}^{T}(n)\right]^{T}
\end{gathered}
$$

where $j=1,2, \ldots, J$ and $L$ is the FIR filter length.

The adaptive filter $\mathbf{W}$, or the ANC controller, has $J$ reference input signals $x_{j}(n)$ that are elements of signal vector $\mathbf{x}(n)$ and generates $K$ secondary signals that are elements of vector $\mathbf{y}(n)$. Therefore, the ANC controller is represented by a $K \times J$ matrix $\mathbf{W}$ ( $K$ rows and $J$ columns), as in Eq. (8)

Each element of the adaptive FIR filter $\mathbf{W}$ has represented by $\mathbf{w}_{k j}(n)$, where $j$ is the reference input index, and $k$ is the secondary source index. The FIR filter has Least Mean Square algorithm, LMS algorithm, to estimate the Mean-Square Error, MSE, for updating the coefficients. This algorithm adjusts the coefficients of the $K \times J$ adaptive filters $\mathbf{w}_{k j}(n)$ in the controller, which can be expressed as in Eq. (9)

$$
\begin{gathered}
\mathbf{W}(n) \equiv\left[\begin{array}{cccc}
\boldsymbol{w}_{11}^{T}(n) & \boldsymbol{w}_{12}^{T}(n) & \ldots & \boldsymbol{w}_{1 j}^{T}(n) \\
\boldsymbol{w}_{21}^{T}(n) & \boldsymbol{w}_{22}^{T}(n) & \cdots & \boldsymbol{w}_{2 j}^{T}(n) \\
\vdots & & \ddots & \vdots \\
\boldsymbol{w}_{k 1}^{T}(n) & \boldsymbol{w}_{k 2}^{T}(n) & \cdots & \boldsymbol{w}_{k j}^{T}(n)
\end{array}\right] \\
\boldsymbol{w}_{k j}(n+1)=\boldsymbol{w}_{k j}(n)+\mu \sum_{m=1}^{M} \boldsymbol{x}_{j}(n) e_{m}(n)
\end{gathered}
$$

where $\mu$ is the step size that determines the stability and the convergence rate of the LMS algorithm.

The output signals $y_{k}(n)$ of ANC systems used to drive $K$ canceling loudspeakers are computed from $\mathbf{x}(n)$ and the adaptive filter matrix $\mathbf{W}$ as in Eqs. (10) and (11)

$$
\begin{aligned}
y_{k}(n)=\sum_{j=1}^{J} y_{k j}(n) & \\
& y_{k j}(n)=\boldsymbol{w}_{k j}^{T}(n) x_{j}(n)
\end{aligned}
$$

where $j=1,2, \ldots, J$ and $k=1,2, \ldots, K$.

In terms of acoustic domain at error sensor $m^{\text {th }}$, determine $d_{m}(n)$ is the desired signal (noise signal in the area), $y_{m}(n)$ is the estimated signal (canceling signal) computed from the adaptive filter (ANC system) and $e_{m}(n)$ is an error signal between $d_{m}(n)$ and $y_{m}(n)$ computed as

$$
e_{m}(n)=y_{m}(n)-d_{m}(n)
$$

If the adaptive filter output $y_{m}(n)$ is identical to the desired signal $d_{m}(n)$. Therefore, when $d_{m}(n)$ and $y_{m}(n)$ are acoustically combined, the residual error is then be

$$
e_{m}(n)=y_{m}(n)-d_{m}(n)=0
$$

which results in perfect cancellation of both sounds of interests based on the superposition principle.

\subsection{Finite difference time domain (FDTD) method}

The FDTD approach has been popularly used and 
employed for many years based on finite-difference approximations to solve simple to complex partial differential equations (PDEs). The close relevancies of the FDTD approach with this research was introduced by Kane S. Yee [27] who applied the FDTD to model electromagnetics (EM), propagating and scattering in 1997. Currently, it has been proved that the FDTD approach is also useful for microwaves, electrodynamics, visible lights, radars, sounds and acoustics propagation etc. This research proposes the use of FDTD approach to realize how acoustics noises propagate in time domain and also to realize how good the ANC system performs when operating in both of space and time domain simultaneously (both acoustic and time domain).

The acoustics wave propagation can be described in term of first order differential equations where the temporal derivative of one field is related to the spatial derivative of another field as in the following Eqs. (14) and (15).

$$
\begin{aligned}
& \frac{\partial p}{\partial t}=-\rho c^{2} \nabla \cdot \boldsymbol{v} \\
& \frac{\partial v}{\partial t}=-\frac{1}{\rho} \nabla p
\end{aligned}
$$

where $p$ represents the scalar sound pressure field, $v$ is the particle velocity vector, $\rho$ is the density of the medium and $c$ is the sound/acoustic speed. In a 3D Cartesian coordinate system, the acoustic wave propagation in the air can be expressed as in Eqs. (16) and (17)

$$
\begin{aligned}
& \frac{\partial p}{\partial t}=-\rho c^{2}\left(\frac{\partial v_{x}}{\partial x}+\frac{\partial v_{y}}{\partial y}+\frac{\partial v_{z}}{\partial z}\right) \\
& \left\{\frac{\partial v}{\partial t}=-\frac{1}{\rho} \frac{\partial p}{\partial x}, \frac{\partial v}{\partial t}=-\frac{1}{\rho} \frac{\partial p}{\partial y}, \frac{\partial v}{\partial t}=-\frac{1}{\rho} \frac{\partial p}{\partial z}\right\}
\end{aligned}
$$

Discretizing those two equations with the finitedifference approximations and staggering gird in time, the solving results can be computed for future fields in terms of known past fields, recursively. Therefore, the acoustically discretizing propagation in both space and time domains can be achieved.

\subsection{Perfectly matched layer (PML) absorbing boundary and conditions}

Numerical modelling of seismic wavefields is important for understanding wave phenomena in complex 2D and 3D media and is essential for full waveform inversion. Due to the restrictions on both memory requirement and computational cost, the model must be limited in size and focused on the area of interest by introducing artificial boundaries.
Therefore, an artificial boundary condition is needed to absorb the energy of the reflections from these artificial boundaries. Two main solutions have been proposed by many researchers in the past: (1) absorbing boundary conditions (ABC) e.g. Clayton and Engquist in 1977 [28], Higdon in 1991 [29] and (2) absorbing boundary layers (PML) e.g. Cerjan et al. in 1985 [30], Bérenger in 1994 [31], Komatitsch and Martin in 2007 [32], Liu and Sen in 2009 [33].

The ABC splits the wave equation into two directions: inside and outside equations using the one-way wave equation method; then, only the outside equation is used on one or two layers outside of the interested area to avoid reflections inwards. The ABC has a good performance when the incident waves are propagating within a certain angle range, especially when the incident waves are close to the direction normal to the boundary. The $\mathrm{ABC}$ has been popular since the early 1970s, and some of the classical $\mathrm{ABCs}$ can be defined up to any desired order; however, the appearance of increasingly highorder derivatives in these $\mathrm{ABCs}$ renders them impractical beyond a certain order, typically 2 or 3 .

The absorbing boundary layers, on the other hand, use many layers to attenuate the artificial reflections gradually; thus, we can greatly reduce artificial reflections with an adequate number of layers. There are three kinds of absorbing boundary layers: (1) the sponge boundary e.g. Cerjan et al. in 1985 [30], (2) the perfectly matched layer (PML) e.g. Bérenger in 1994 [31] and Chew and Liu in 1996 [34] and (3) the hybrid absorbing boundary layer proposed by Liu and Sen in 2012 [35].

The most famous PML proposed by Bérenger in 1994 [31] applies a completely new mechanism to avoid apparent artificial reflections. Bérenger's PML introduces physical attenuations to the wave equation. The PML modifies the partial derivatives in the wave equation using complex coordinate stretching by introducing an imaginary part associated with an attenuation factor. Complex coordinate stretching is well known for viscoelastic media to understand the nature of the intrinsic attenuation for wave propagation; thus, the PML presents a nice rule in designing the optimal attenuation coefficients by tuning the attenuation factor. Many modifications had been proposed based on Bérenger's PML concepts. Kristek finally made a summary review about the variations of the PML research: the split/unsplit, classical/convolutional and general/special PML formulations, which can help the new engineers understand many classifications of different PML formulations, in 2009 [36].

Consider the acoustics wave propagation using 


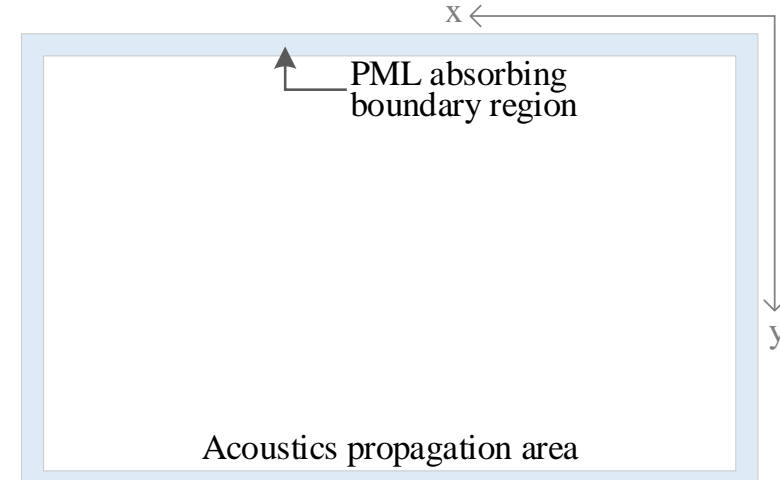

Figure. 5 The acoustics propagation area with the PML absorbing

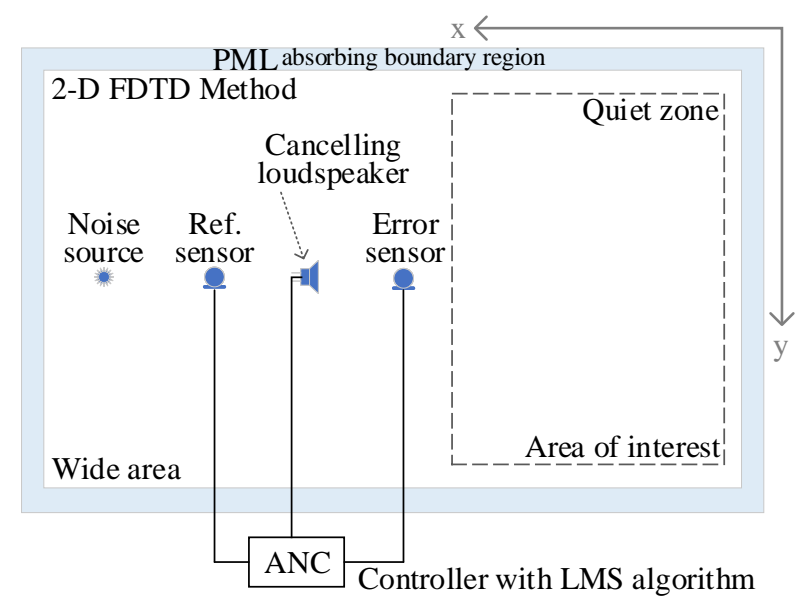

Figure. 6 A two-dimension wide-area virtual environment space modeling and configuration

the FDTD approach, at the end of the girds without absorbing boundary layers, the acoustics wave reflection will be occurred. To eliminate the acoustics wave reflection, in this paper, we applied the main concept from Berenger's PML model with 2D scalar wave equations proposed by Liu and Tao [37] in 1997 and Hustedt [38] in 2004. We then did a slight modification to combine a two-dimension FDTD governing acoustic equations with PML technique. The modification of a 2D-FDTD scalar wave equations with PML technique can be rewritten as

$$
\begin{gathered}
\left\{\begin{array}{c}
\frac{\partial p_{x}}{\partial t}+D(x) p_{x}=-\rho c^{2} \frac{\partial v_{x}}{\partial x} \\
\frac{\partial p_{y}}{\partial t}+D(y) p_{y}=-\rho c^{2} \frac{\partial v_{y}}{\partial y}
\end{array}\right\} \\
\left\{\begin{array}{c}
\frac{\partial v_{x}}{\partial t}+D(x) v_{x}=-\frac{1}{\rho} \frac{\partial(p)}{\partial x} \\
\frac{\partial v_{y}}{\partial t}+D(y) v_{y}=-\frac{1}{\rho} \frac{\partial(p)}{\partial y}
\end{array}\right\} \\
p=p_{x}+p_{y}
\end{gathered}
$$

where $D(x)$ and $D(y)$ are the absorbing boundary coefficients designed to attenuate the reflection zone [39]. The absorbing boundary coefficients can be model as

$$
\left\{\begin{array}{l}
D(x)=\frac{3 v}{2 L} \log \left(\frac{1}{R}\right)\left(\frac{x}{L}\right)^{2} \\
D(y)=\frac{3 v}{2 L} \log \left(\frac{1}{R}\right)\left(\frac{y}{L}\right)^{2}
\end{array}\right\}
$$

where $v$ is the velocity of acoustics at $\mathrm{x}-\mathrm{y}, L$ is the width of the absorbing layer and $R$ is the theoretical reflection coefficient of the split PML [39-40].

Therefore, we choose to use this integration of the 2D FDTD approach with the modified PML technique throughout this research to ensure the accuracy and performance of the proposed ANC system; and to show the visualization of the propagation filed, in all simulations cases.

\section{The proposed methodology}

This section will elaborate how we can create the model to represent the actual wide-area environment in simulating the acoustic propagation space, how to integrate the FDTD approach with PML technique to visualize the sound pressure level and also brief details of the feedforward ANC system with LMS algorithm used in this research.

\subsection{Wide-area model and system configurations}

In this paper, a wide-area actual environment will be setup as a two-dimension propagation space as can be seen in Fig. 6. The PML absorbing boundary has been set to cover the entire space to ensure the minimum to zero acoustic reflection. A single noise source will be placed inside this space (locations may vary for different simulating purposes).

A single-channel feedforward ANC system mentioned in the previous section will mainly be used in this modeling and simulation for simplicity. ANC sensors and actuators (loudspeakers) will be setup inside the space while the ANC controller will be located outside of this space as in actual environment. The desired quiet zone (or silent zone) will be determined as a rectangular area inside this space. A simplified diagram of the proposed model and configurations can be seen in Fig. 7.

\subsection{Discretized FDTD with PML boundary}

One main goal of this research is to visualize and combine the acoustic domain to the ANC operation in time domain. To combine the acoustics-domain propagation space to the ANC operation in time 


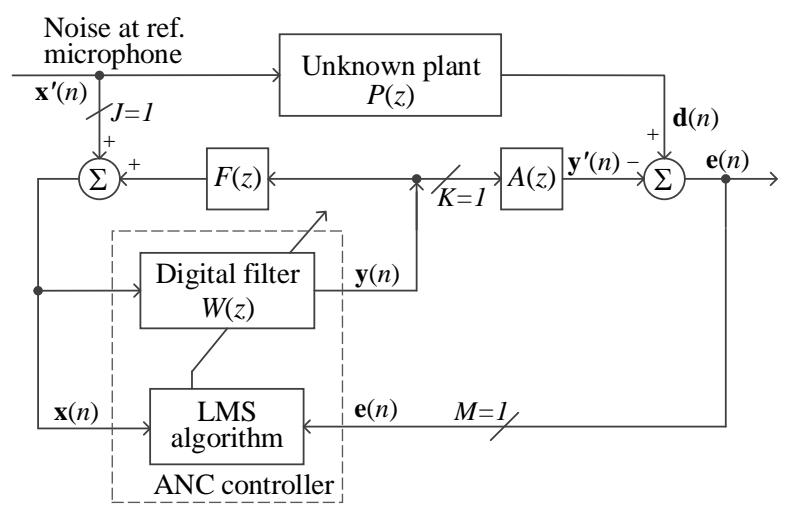

Figure. 7 A simplified diagram of the proposed feedforward ANC system configurations in z-domain

domain, the discretization of the FDTD approach with PML absorbing boundary to determine sound pressure levels $\left(p_{x}, p_{y}\right)$ and the particle velocities $\left(\mathbf{v}_{x}\right.$, $\mathbf{v}_{y}$ ) shall be performed first. It should be noted here that, sampling numbers will have an influence on the acoustics wave propagation calculation significantly. A two-dimension space with grids of sound pressure level $(p)$ and velocity (v) will be explored here. The first-order forward approximation will be employed to update the time steps in this process.

$$
\begin{gathered}
\left(\frac{p_{x}(i, j, n+1)-p_{x}(i, j, n)}{\Delta t}\right) \\
+D(x)\left(\frac{p_{x}(i, j, n+1)-p_{x}(i, j, n)}{2}\right) \\
=-\rho c^{2}\left(\frac{\boldsymbol{v}_{x}(i+0.5, j, n)-\boldsymbol{v}_{x}(i-0.5, j, n)}{\Delta x}\right) \\
\left(\frac{p_{y}(i, j, n+1)-p_{y}(i, j, n)}{\Delta t}\right) \\
+D(y)\left(\frac{p_{y}(i, j, n+1)-p_{y}(i, j, n)}{2}\right) \\
=-\rho c^{2}\left(\frac{\boldsymbol{v}_{y}(i, j+0.5, n)-\boldsymbol{v}_{x}(i, j-0.5, n)}{\Delta y}\right) \\
\left(\frac{\boldsymbol{v}_{x}(i+0.5, j, n+1)-\boldsymbol{v}_{x}(i+0.5, j, n)}{\Delta t}\right) \\
+D(x)\left(\frac{\boldsymbol{v}_{x}(i+0.5, j, n+1)-\boldsymbol{v}_{x}(i+0.5, j, n)}{2}\right) \\
=-\frac{1}{\rho}\left(\frac{p(i+1, j, n)-p(i, j, n)}{\Delta x}\right) \\
\left(\frac{\boldsymbol{v}_{y}(i, j+0.5, n+1)-\boldsymbol{v}_{y}(i, j+0.5, n)}{\Delta t}\right) \\
+D(y)\left(\frac{\boldsymbol{v}_{x}(i+0.5, j, n+1)-\boldsymbol{v}_{x}(i+0.5, j, n)}{2}\right) \\
=-\frac{1}{\rho}\left(\frac{p(i, j+1, n)-p(i, j, n)}{\Delta y}\right)
\end{gathered}
$$

where $\Delta x$ and $\Delta y$ are the grid sizes in the $x$ - $y$ axis, $i$ and $j$ are spatial indices of the grid and $n$ is the time step.

From Eqs. (22-25), the discretized sound pressure $p_{x}$ and $p_{y}$ and the velocity $\mathbf{v}_{x}$ and $\mathbf{v}_{y}$ can be derived as follows.

$$
\begin{gathered}
p_{x}(i, j, n+1)=\left(\frac{2-D(x) \Delta t}{2+D(x) \Delta t}\right)\left(p_{x}(i, j, n)\right)- \\
\frac{\rho c^{2}}{\Delta x}\left(\frac{2 \Delta t}{2+D(x) \Delta t}\right)\left(\boldsymbol{v}_{x}(i+0.5, j, n)-\boldsymbol{v}_{x}(i-\right. \\
0.5, j, n))
\end{gathered}
$$

$$
\begin{aligned}
& p_{y}(i, j, n+1)=\left(\frac{2-D(y) \Delta t}{2+D(y) \Delta t}\right)\left(p_{y}(i, j, n)\right)- \\
& \frac{\rho c^{2}}{\Delta y}\left(\frac{2 \Delta t}{2+D(y) \Delta t}\right)\left(\boldsymbol{v}_{y}(i, j+0.5, n)-\boldsymbol{v}_{y}(i, j-\right. \\
& 0.5, n)) \\
& \boldsymbol{v}_{x}(i+0.5, j, n+1) \\
& =\left(\frac{2-D(x) \Delta t}{2+D(x) \Delta t}\right) \boldsymbol{v}_{x}(i \\
& +0.5, j, n)-\ldots \\
& \ldots \frac{1}{\rho \Delta x}\left(\frac{2 \Delta t}{2+D(x) \Delta t}\right)(p(i+1, j, n)-p(i, j, n)) \\
& \boldsymbol{v}_{y}(i, j+0.5, n+1) \\
& =\left(\frac{2-D(y) \Delta t}{2+D(y) \Delta t}\right) v_{y}(i, j+0.5, n)- \\
& \frac{1}{\rho \Delta y}\left(\frac{2 \Delta t}{2+D(y) \Delta t}\right)(p(i, j+1, n)-p(i, j, n))
\end{aligned}
$$

The computational process starts from computing $x$-axis propagation, $p_{x}$ at $n+1$ from $\mathbf{v}_{x}$ at $n$ and the previous $p_{x}$. Likewise, $\mathbf{v}_{x}$ at $n+1$ can then be computed from $p_{x}$ at $n$ and the previous $p$. Repeat those $p_{x}$ computational steps by increasing $n$ by one. For the $y$-axis propagation, same process shall be done here. As a result, the simulation for acoustics wave propagation can be done in the spatial domain and time.

\subsection{Feedforward ANC with LMS algorithm}

The ANC system used in this work is a slight modification from a general ANC architecture previously mentioned in section 2.2 (Fig. 3 and Fig. 4).

By setting $j=1$ and $k=1$, at a reference sensor, $\mathbf{x}(n)$ and the cancelling loudspeaker signal, $y(n)$, can then be derived by Eqs. (30) and (31) below. This is called a single-channel feedforward ANC system. The LMS algorithm to update the coefficients is still be used here. The adaptive filter, $\mathbf{w}(n)$, which includes the 


\begin{tabular}{|c|c|c|}
\hline \multicolumn{3}{|c|}{ Real-Time ANC simulations using FDTD approach With PML boundary. } \\
\hline \multicolumn{3}{|c|}{ ANC design: single channel, adaptive filter type, filter length, algorithm } \\
\hline \multicolumn{3}{|c|}{$\begin{array}{c}\text { FDTD design: 2-D propagation dimension, grid width, finite difference } \\
\text { approximations orders, time steps. }\end{array}$} \\
\hline \multicolumn{3}{|c|}{ PML design: PML width, PML location, reflection coefficient. } \\
\hline \multicolumn{3}{|c|}{ Input: noise signal, ANC device locations. } \\
\hline \multicolumn{3}{|c|}{ Computational procedure: } \\
\hline \multicolumn{3}{|c|}{ Start define simulation time (number of time steps) } \\
\hline \multicolumn{3}{|c|}{ 1. noise pass through FDTD simulation as sound pressure. } \\
\hline \multicolumn{3}{|c|}{ 2. compute noise propagation for each time step. } \\
\hline \multicolumn{3}{|c|}{ 3. ANC sensor takes the sound outputs computed from FDTD. } \\
\hline \multicolumn{3}{|c|}{ 4. ANC computes anti-noise and sends to loudspeaker. } \\
\hline \multicolumn{3}{|c|}{ 5. Error sensor picks the residual noise and sends back to ANC. } \\
\hline \multicolumn{3}{|c|}{ 6. Propagate noise and anti-noise as sound pressure level. } \\
\hline \multicolumn{3}{|c|}{ End repeat until the last time step. } \\
\hline \multicolumn{3}{|c|}{ Final Outcome: real-time noise and anti-noise (from ANC) propagation. } \\
\hline \multicolumn{3}{|c|}{ Figure. 8 The simulation pseudo process } \\
\hline \multicolumn{3}{|c|}{ Table 1. Simulation parameters } \\
\hline Symbol & Definition & $\begin{array}{l}\text { Parameter } \\
\text { Value }\end{array}$ \\
\hline$C$ & speed of noise & $340 \mathrm{~m} / \mathrm{s}$ \\
\hline$P$ & density of air & $1.21 \mathrm{~kg} / \mathrm{m}^{3}$ \\
\hline$X$ & number of nodes in $\mathrm{x}$-direction & 460 \\
\hline$Y$ & number of nodes in y-direction & 335 \\
\hline$x-P M L$ & $\begin{array}{l}\text { number of nodes in PML } \\
\text { regions in } \mathrm{x} \text {-direction }\end{array}$ & 20 \\
\hline$y-P M L$ & $\begin{array}{l}\text { number of nodes in PML } \\
\text { regions in y-direction }\end{array}$ & 20 \\
\hline$\Delta x, \Delta y$ & $\begin{array}{l}\text { distance between nodes, or the } \\
\text { size of the grids }\end{array}$ & $0.024 \mathrm{~m}$ \\
\hline$\Delta t$ & propagation time steps & $5 \times 10^{-5}$ sec. \\
\hline$R$ & theoretical reflection coefficient & $10^{-3}-10^{-7}$ \\
\hline$p_{0}$ & reference sound pressure in air & $20 \mu \mathrm{Pa}$ \\
\hline$f_{s}$ & frequency sampling & $2 \times 10^{-4} \mathrm{~Hz}$ \\
\hline Noise & pure sine wave & $1 \mathrm{kHz}$ \\
\hline M & filter length (FIR filter) & 128 \\
\hline$\mu$ & step size & 0.001 \\
\hline
\end{tabular}

primary and secondary acoustic paths can also be simplified as in Eq. (32).

$$
\begin{gathered}
\boldsymbol{x}(n)=[x(n), x(n-1), \ldots, x(n-1+L)]^{T} \\
y(n)=\boldsymbol{w}^{T}(n) \boldsymbol{x}(n) \\
\boldsymbol{w}(n+1)=\boldsymbol{w}(n)+\mu \boldsymbol{x}(n) e(n)
\end{gathered}
$$

\subsection{Simulation procedures of proposed analysis}

To visualize the sound pressure propagations in a wide-area virtual environment, using the FDTD approach with PML absorbing boundaries and a single-channel feedforward ANC system with LMS algorithm, the simulation procedures shown in Fig. 8 must be followed.

From the procedure shown in Fig. 8, the grid width, $\Delta x, \Delta y$ should be less than the shortest wavelength of the incoming noise to accurately propagate the noise pressure level in the air without oversampling.

$$
\frac{c \Delta t}{\Delta x} \leq \frac{1}{\sqrt{2}}
$$

The stability of the FDTD computation will be assured, if and only if the courant factor, $c \Delta t / \Delta x$, is less or equal to $1 / \sqrt{ } \mathrm{n}$, where $n$ is the FDTD dimension so that the two-dimensional FDTD equation can be expressed as in Eq. (33).

It should be noted here that the key correlation between two domains (acoustic and electrical) is the sampling rate of any noise source. In this case, the sampling rate of at least $f_{s}=1 / \Delta t$ shall be used here for smooth performance.

\section{Simulation results}

In this section, four different simulation results from the proposed model and methodology will be presented in several aspects. We start from observing the acoustics filed of FDTD propagation combining with PML technique and not activating the ANC just yet. The second simulation result presents the sound pressure fields and noise attenuation capabilities in term of the average sound pressure level (SPL) when integrating the ANC operation with various ANC device locations. The third simulation result presents the ANC performance effects when moving each ANC device location. And the last simulation result shows the acoustic filed and an attenuation capability when installing ANC devices in optimum locations. In this section, all the FDTD-PML-ANC simulation parameters are set to be the same for consistency. Table 1 below clarifies all the setting parameters to be used throughout these simulating sections.

\subsection{Sound pressure field propagation using FDTD approach with and without PML technique}

A goal for the first simulation is to visualize the acoustics propagation in term of sound pressure field when applying the discretized FDTD set of equations in a wide-area virtual environment. The space of simulation is a rectangular area, size of 9x12 meters. A noise source ( $1 \mathrm{kHz}$ sinusoidal wave, $\lambda=0.34$ meter and amplitude of $2 \mathrm{~Pa}$.) is set to be at coordinate 


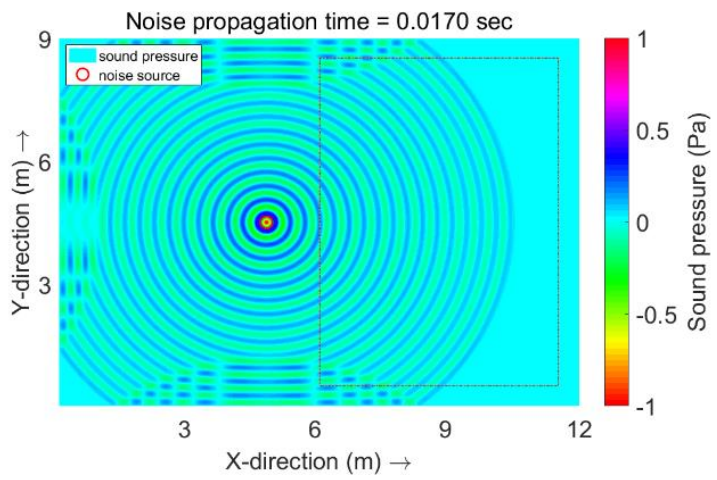

(a)

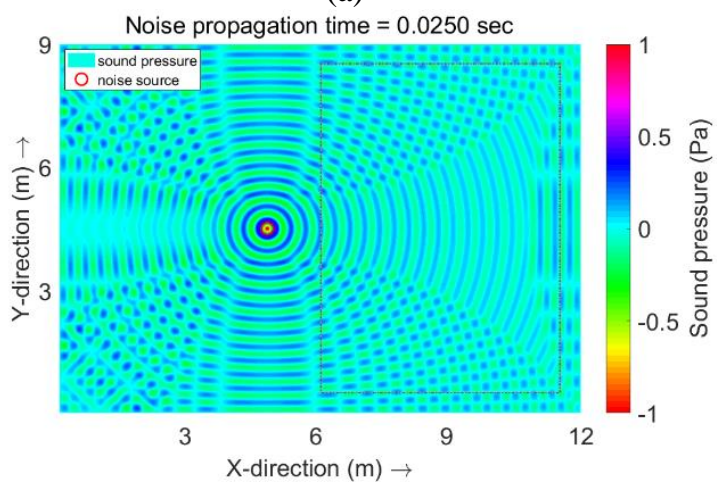

(b)

Figure. 9 The sound pressure propagation fileds from a signle noise source without PML technique: (a) at $\mathrm{t}=0.0170$ second and $(\mathrm{b})$ at $\mathrm{t}=0.0250$ second

( $x=4.80 \mathrm{~m}, y=4.48 \mathrm{~m}$ ). A grid for FDTD computation equals to $0.024 \mathrm{~m}$ for both $x$ and $y$ directions. The PML staggering layer is set to be 20 .

Fig. 9 (a) and Fig. 9 (b) demonstrate the acoustics propagation field (at $t=0.0170$ second and $t=0.0250$ second, respectively) in terms of sound pressures from a single noise source in a virtual wide-area environment using the FDTD approach. Without applying the PML technique, the artificial reflection waves clearly exist in the propagation field and appear at every boundary of the propagation space. These artificial reflections could cause the inaccuracy and performance drop when integrating the ANC system to the simulation. Therefore, it is vital to apply the PML technique for eliminating those artificial reflections to make it as close as possible to the widearea virtual environment. By adding PML technique to FDTD simulations, with the same configuration as in Fig.9, it can clearly be seen from Fig.10(a) and Fig.10(b) that there is no reflection existed in the propagation space. The results from these two figures confirm that the PML absorbing boundary efficiently helps in reducing the artificial reflections. This PML technique will be beneficial to ensure the accuracy and stability when integrating the ANC system to the simulation.

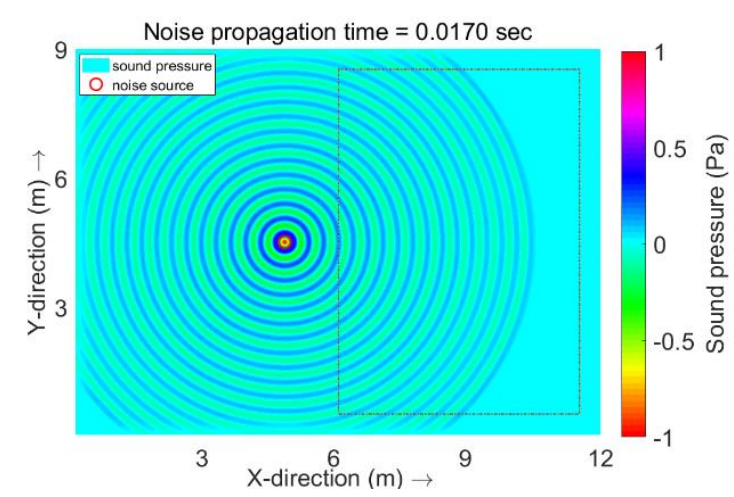

(a)

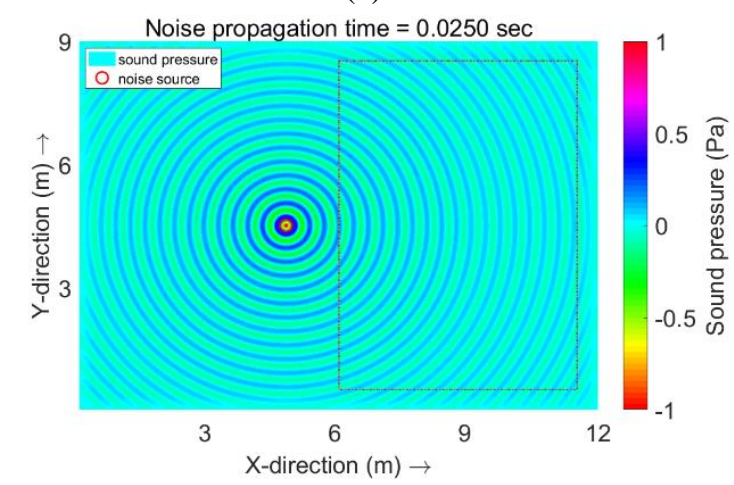

(b)

Figure. 10 The sound pressure propagation fileds from a signle noise source with PML technique: (a) at $\mathrm{t}=0.0170$ second and (b) at $t=0.0250$ second

It should be mentioned that for a global noise attenuation analysis, we may consider every point of noise attenuation in this space; however, in the actual wide-area environment, the noise source, the ANC devices and the area of interest (quiet zone) are most likely located in different areas. Therefore, the quiet zone throughout every simulation will be set inside the propagation space and represented by a dashed rectangular area on the middle-right of the space with the size of 5x8 meters, as shown in Fig.10 and Fig.11.

\subsection{Integrating the ANC system simulation}

In this section, the acoustics propagation field from the FDTD approach and PML technique will be integrated with a single-channel feedforward ANC system. Two randomly picked ANC device locations will be chosen and evaluated. Our hypothesis is that different ANC device locations will give us different results in terms of acoustic propagation field and noise attenuation (average SPL in $\mathrm{dB}$ ) in the desired quiet zone. A sinusoidal signal $(1 \mathrm{kHz})$ with the amplitude of $1 \mathrm{~Pa}$. will be defined as a noise source in this simulation. The quiet zone in this simulation is defined as a dashed-line rectangular area with the size of 5.5x8.0 m (in Fig.11(a), (b) and (c)).

Fig. 11 (a) shows the base-line configuration for 


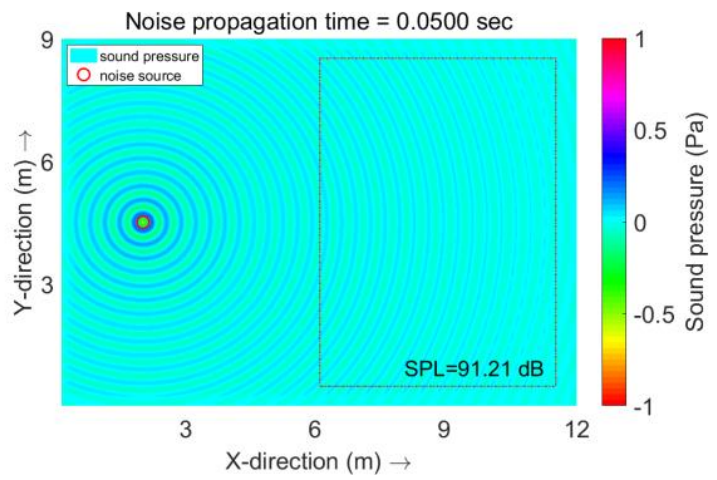

(a)

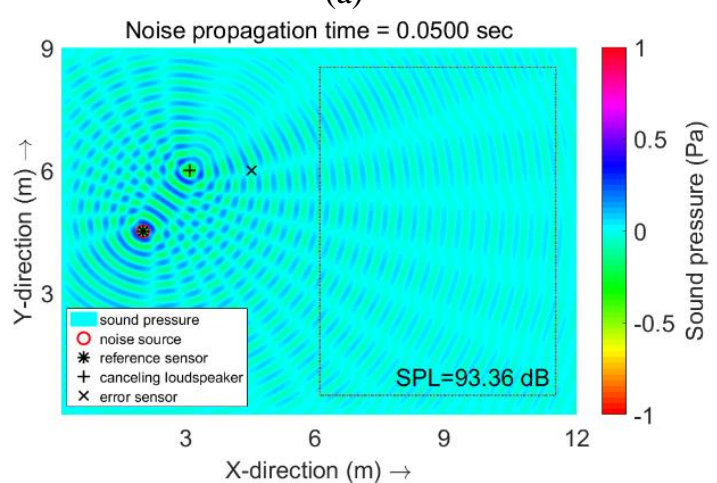

(b)

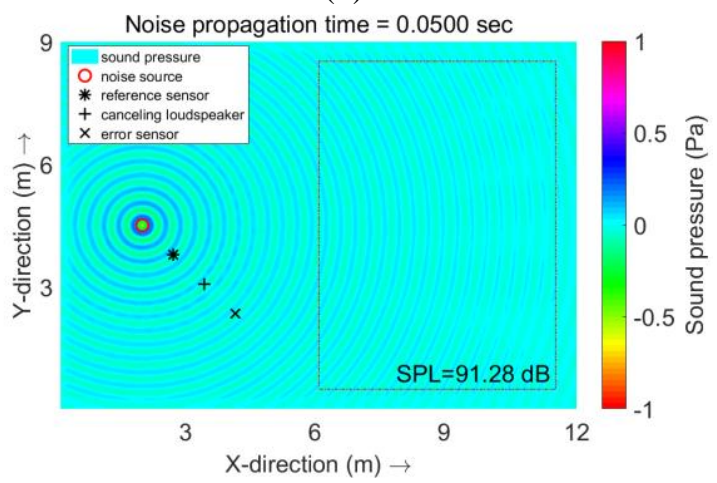

(c)

Figure. 11 The acoustic propagation filed using FDTD and PML approach with the ANC system operating in a wide-area virtual environment with different locations of the ANC devices: (a) base line, (b) ANC devices position $\# 1$ and (c) ANC devices position \#2

the acoustic propagation with no ANC installing. The noise source is set at coordinate $(x=1.92, y=4.52)$. We can visualize the sound pressure field and compute the average SPL in the quiet zone as $91.21 \mathrm{~dB}$. The second configuration, shown in Fig.11(b), the noise source and the reference sensor are set at the same coordinate $(x=1.92, y=4.52)$, the loudspeaker is set to be at $(x=3.0, y=6.0)$ and the error sensor is set to be at $(x=4.44, y=6.0)$. This setting clearly shows different acoustics filed and yields the noise attenuation 93.36 $\mathrm{dB}$ in a quiet zone. For the third configuration, shown in Fig.11(c), a reference sensor is set at coordinate $(x=2.64, y=3.80)$, the cancelling loudspeaker is set to

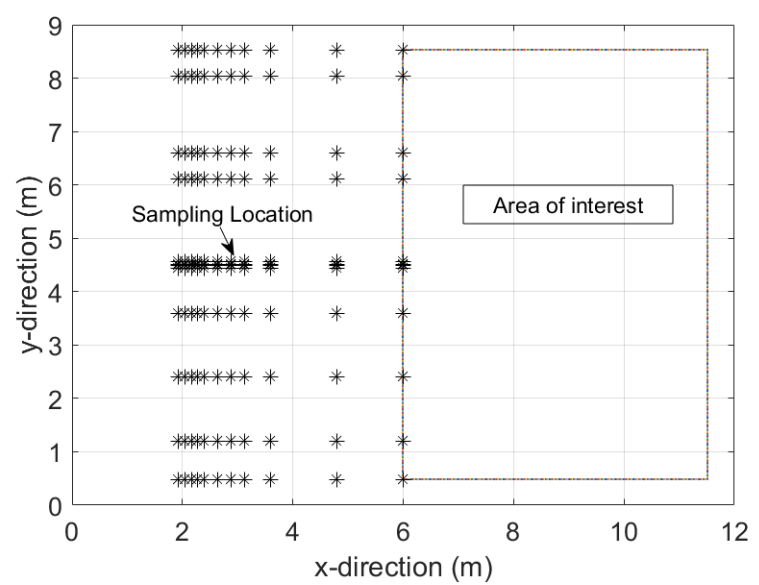

Figure. 12 ANC devices sampling locations to be used in this section's simulations

be at $(x=3.36, y=3.08)$ and the error sensor is set to be at $(x=4.08, y=2.36)$. This setting yields the noise attenuation $91.28 \mathrm{~dB}$ in a quiet zone. It can be seen from this simulation that the ANC device locations are super important. Different ANC device locations result in different noise attenuation capabilities.

\subsection{SPL effects on a variety of sampling locations for each ANC device}

The main goal for this simulation is to analyse how the proposed ANC shall perform when moving each of its device locations. The average SPL (in $\mathrm{dB}$ ) of the remaining noise in a quiet zone will be used as the main indicator. All simulations in this section, the noise source location will be fixed at $(x=2.4, y=4.5)$. Three cases of the simulations will be considered: (1) the average SPL effects when varying the reference microphone locations, (2) the average SPL effects when varying the cancelling loudspeaker locations and (3) the average SPL effects when varying the error sensor locations.

The sampling and moving locations for the ANC devices are shown in Fig. 12. The distance between location was randomly picked as a multiplication of a noise signal wavelength $(n \lambda)$. In these simulations, the $11 \times 12$ sampling grids were selected (total of 132 grid locations). the noise source amplitude is set to be $1 \mathrm{~Pa}$. The average SPL effects on each ANC device moving locations will be evaluated and plotted. It should be noted here that the gird size for computing the average SPL in the quiet zone is set to be 10,000 points (100x100). Fig. 13 illustrates the SPL contour (in $\mathrm{dB}$ ) of the remaining noises in a quiet zone when moving the ANC device related to each sampling location shown in Fig. 12 for three configurations. The lower point in SPL contour, the better ANC performs. 


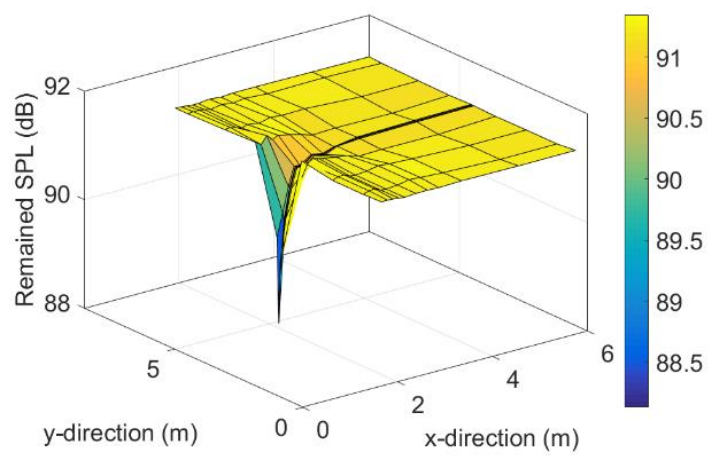

(a)

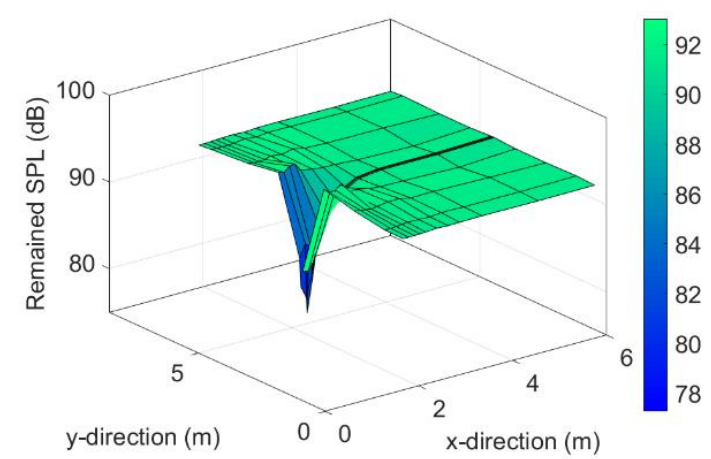

(b)

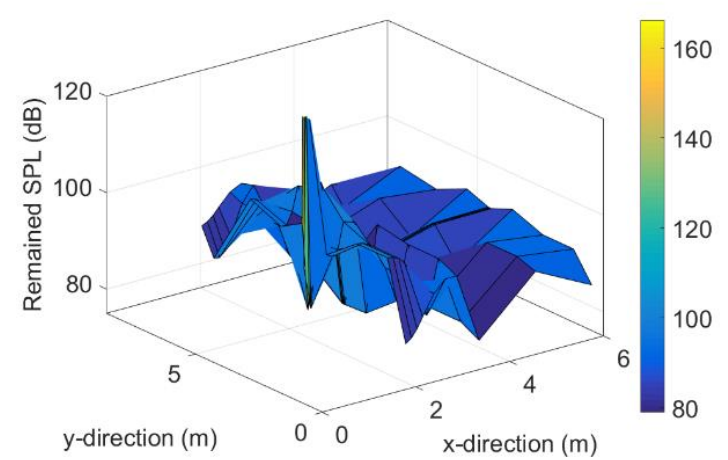

(c)

Figure. 13 Three SPL contours in the quiet zone: (a) location variations of a reference microphone, (b) location variations of a cancelling loudspeaker and (c) location variations of the error microphone

Fig. 13 (a) illustrates the remaining noise SPL contour when moving a reference microphone's locations. Finding is that the closer reference microphone to the noise source, the better noise attenuation. The best ANC performance in this case occurred when the reference microphone is located closet coordinate as the noise source. Fig. 13 (b) demonstrates the SPL contour for each cancelling loudspeaker moving location. Finding is that the closer loudspeaker to the noise source tends to yield better ANC performance. The best ANC performance in this case occurred when the loudspeaker is horizontally aligned with the noise source and $0.35 \lambda$

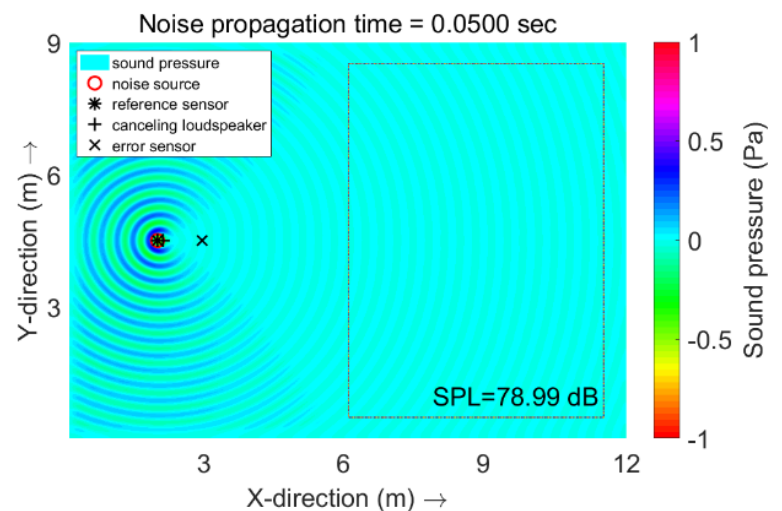

Figure. 14 Acoustic propagation field and the remaining noise SPL in a desired quiet zone from the optimum ANC device locations

away from the noise source. Fig. 13 (c) exemplifies the SPL contour when moving the error microphone locations. Finding is that the error microphone location significantly effects the ANC performance. Unlike the first two cases, the best ANC performance in this case occurred when the error microphone is horizontally aligned with noise source and $2.8 \lambda$ away from the noise source.

\subsection{Acoustic filed and noise attenuation capability when ANC devices in optimum locations.}

The optimum locations for installing each of the ANC device that are a reference sensor, a cancelling loudspeaker and an error sensor has carefully been picked based on previous simulation results. Using the proposed FDTD approach and PML technique with a feedforward ANC system and LMS algorithm, the acoustic propagation and the noise attenuation capability in a quiet zone can be achieved here.

For this simulation, a noise source and a reference microphone, both are set to be at the same coordinate $(x=1.92, y=4.52)$, a cancelling loudspeaker is set to be at $(x=2.04, y=4.52)$ and the error sensor is set to be at $(x=2.88, y=4.52)$. The acoustic propagation filed can be visualized here. The attenuation capability for the remaining noise SPL in the quiet zone is happened to be $78.99 \mathrm{~dB}$ (comparing to $91.21 \mathrm{~dB}$ with no ANC installation). The acoustic propagation simulations in the space are shown in Fig.14.

\section{Conclusions}

From the simulation results, many points of conclusions can be made here. Firstly, the acoustic wave propagation in a wide-area space can clearly be visualized and analysed using the proposed FDTD approach. The PML absorbing boundary condition was integrated to the FDTD propagation space to eliminate unwanted artificial reflections in all space 
borders. The combination of these two techniques helps in making more accurate model of a wide-area virtual environment.

Secondly, by discretizing the FDTD equations with PML absorbing conditions in acoustic domain, the integration with a single-channel feedforward ANC system with LMS algorithm set of equations in a time domain can be achieved. A variety of ANC system operations in a wide-area virtual environment can easily and clearly be observed and visualized in term of the acoustic propagation fields. The ANC system performance can also be directly computed in term of a noise attenuation capability using a unique indicator called an average sound pressure level (SPL) focusing on the area of interest (or a desired quiet zone).

Thirdly, in a general ANC system research, not only the primary path effect shall be considered, but the secondary path and feedback path effects shall also be evaluated to ensure the system accuracy and effectiveness. The secondary path and feedback path effects are usually counted as two of the most challenging issues in the ANC research field. It worth mentioning here that the proposed integration method in this work already includes those two-path effects in considerations.

And lastly, by using the proposed discretizing FDTD and PML technique and a feedforward ANC with LMS algorithm, the acoustic propagation field and the noise attenuation capability computed at any point in a wide-area virtual space will be available. That means we can move a noise source or even any of ANC device locations to evaluate how the ANC system performance changes. Determining optimum locations for each ANC device is another positive outcome that will be available with the proposed model and analysis. It is apparent from the previously shown simulation results that ANC device's location change would directly affect the propagation field and the average SPL in the quiet zone. The optimum locations for each of the ANC device in this research have been identified and already shown in the previous section. Using the proposed model and approach, the noise attenuation in a desired quiet zone could be reduced by the maximum of 13.39 percent in term of the average SPL in the zone of interest. It should be noted here that, in this research, we neglected the acoustic reflection from the ground and the acoustic effect from the natural wind that could also make some impacts to the system performance.

\section{Future work}

The proposed integration model between the ANC system and the FDTD with PML propagation technique is an intermediate step of a wide-area ANC analysis. The simulation results from this research confirm the efficiency of the proposed methodology in many ways: modeling, visualizing, and analyzing a single-channel feedforward ANC system in a widearea virtual environment. However, there are always some rooms for improvements. Additional advanced ANC algorithms like FXLMS algorithm or others could be considered. A more-complex multi-channel feedforward ANC architecture could also be applied based on this proposed model and configuration. Various optimization techniques could potentially be considered in determining the optimum locations for each of the ANC device locations in a more-complex space. These topics should be good guidelines for those who are interested in further research in this wide-area ANC analysis.

\section{Acknowledgment}

The authors would like to thank the Research and Researchers Industries Department (RRI Funding), Thai Government, for fully support in beneficial information and sponsorship. This work is under a funding contract PHD-58I0087 from RRI.

\section{References}

[1] P. Lueg, "Process of silencing sound oscillations", U.S. Patent 2043 416, Jun. 9, 1936.

[2] B. Widrow, J. R. Glover, JR., J. M. Mccool, J. Kaunitz, C. S. Williams, R. H. Hearn, J. R. Zeidler, E. Dong, and R. C. Goodlin, "Adaptive noise cancelling: Principles and applications", In: IEEE Proc., Vol. 63, No. 12, pp. 1692-1716, 1975.

[3] S. M. Kuo and D. R. Morgan, "Active noise control: a tutorial review", In: IEEE Proc., Vol. 87, No. 6, pp. 943-973, 1999.

[4] D. C. Chang and F. T. Chu, "Feedforward active noise control with a new variable tap-length and step-side filtered-x LMS algorithm", IEEE/ACM Trans. on Audio, Speech, and Language Processing, Vol. 22, No. 2, pp. 542-555, 2014.

[5] I. T. Ardekani and W. H. Abdulla, "Active noise control in three dimensions", IEEE Trans. on Control Systems Technology, Vol. 22, No. 6, pp. 2150-2159, 2014.

[6] S. M. Kuo, S. Mitra, and W. S. Gan, "Active noise control system for headphone applications", IEEE Trans. on Control Systems Technology, Vol. 14, No. 2, pp. 331-335, 2006. 
[7] L. Sujbert and A. Szarvas, "Noise-canceling office chair with multiple reference microphones", Appl. Sci., Vol. 8, No. 1702, doi:10.3390/app809172, 2018.

[8] A. Chraponska, S. Wrona, J. Rzepecki, K. Mazur, and M. Pawelczyk, "Active structural acoustic control of an active casing placed in a corner", Appl. Sci., Vol. 9, No. 1059, doi:10.3390/app9061059, 2019.

[9] D. Graupe and A. Efron, "An adaptive-controlbased noise cancellation approach", In: Proc. of IEEE Sym., Singapore, 1991.

[10] A. J. Efron and L. C. Han, "Wide-area adaptive active noise cancellation", IEEE Trans., Vol. 41, No. 6, pp. 405-409, 1994.

[11] G. Gäbel, J. Millitzer, H. Atzrodt, and S. Herold, "Development and implementation of a multichannel active control system for the reduction of road induced vehicle interior noise", Actuators, Vol. 7, No. 52, doi:10.3390/act7030052, 2018.

[12] L. Liu, Y. Li, and S. M. Kuo, "Feed-forward active noise control system using microphone array”, IEEE Journal, Vol. 5, No. 5, pp. 946-952, 2018.

[13] K. Iwai, S. Hase, and Y. Kajikawa, "Multichannel feedforward active noise control system with optimal reference microphone selector based on time difference of arrival", Appl. Sci., Vol. 8, No. 2291, doi:10.3390/app8112291, Nov. 2018.

[14] J. He, B. Lam, D. Shi, and W. S. Gan, "Exploiting the underdetermined system in multichannel active noise control for open windows", Appl. Sci., Vol. 9, No. 390, doi:10.3390/app9030390, 2019.

[15] L. Ying, J. Wang, Q. Liu, and D. Wang, "Application study of adaptive tracking algorithm in active noise control system of transformer", Appl. Sci., Vol. 9, No. 2693, doi:10.3390/app9132693, 2019.

[16] C. Zhang, M. Qin, H. Zou, and X. Qiu, "Secondary source and error sensing strategies for the active control of sound transmission through a small opening", Elsevier, No. 114973, https://doi.org/10.1016/j.jsv.2019. 114973, 2019.

[17] M. W. Munir and W. H. Abdulla, "On FxLMS scheme for active noise control at remote location", IEEE Access, Vol. 8, pp. 21407121472, 2020.

[18] Y. Maeno, Y. Mitsufuji, P. N. Samarasinghe, N. Murata and T. D. Abhayapala, "Sphericalharmonic-domain feedforward active noise control using sparse decomposition of reference signals from distributed sensor arrays", IEEE Trans., Vol. 28, pp. 656-670, 2020.

[19] F. Ma, W. Zhang, and T. D. Abhayapala, "Active control of outgoing broadband noise fields in rooms", IEEE Trans., Vol. 28, pp. 529539, 2020.

[20] H. Sun, T. D. Abhayapala, and P. N. Samarasinghe, "A realistic multiple circular array system for active noise control over 3D space", IEEE Trans., Vol. 28, pp. 3041-3052, 2020.

[21] A. Szarvas and L. Sujbert, "Efficiency Testing of Active Noise Control by Acoustic Field Modeling", Periodica Polytechnica Elec. Eng. And Com. Sci., Vol. 59, No. 4, doi: 10.3311/PPee .8453, pp. 147-159, 2015.

[22] K. Sankaran, "Are you using the right tools in computational electromagnetics?", Wiley, doi.org/10.1002/eng2.12041, 2019.

[23] D. Orlis, M. Giouvanakis, C. Sevastiadis and G. Papanikolaou, "Simulation of active low frequency noise control in closed spaces using the FDTD method", In: Euronoise Conf. Proc., Heraklion, Crete, Greece, pp.929-934, 2018.

[24] C. Sookpuwong and C. C. Inwai, "Performance comparisons between a single-channel feedforward ANC system and Single-channel feedback ANC system in a noisy-environment classroom", In: Conf. Proc. of ISEIM, Nagoya, JAPAN, pp.203-206, 2017.

[25] C. Sookpuwong and C. C. Inwai, "A multichannel feedforward ANC with FXLMS algorithm for aviation-noise suppression", In: IEEE Conf. Asilomar, Pacific Grove, CA, USA, pp.1374-1378, 2019.

[26] C. Sookpuwong and C. C. Inwai, "A multichannel Feedforward ANC system using a novel FXLMS algorithm in solving classroom aviation-noise problems", IJEECT, Vol. 8, No.6, 2019.

[27] K. S. Yee and J. S. Chen, "The finite-difference time-domain (FDTD) and the finite-volume time-domain (FVTD) methods in solving Maxwell's equations", IEEE Trans. on antennas and propagation, Vol. 45, No.3, pp. 354-363, 1997.

[28] R. Clayton and B. Engquist, "Absorbing boundary conditions for acoustic and elastic wave equations", Bull. Seismol. Soc. Am., Vol. 67, pp. 1529-40, 1977.

[29] R. Higdon, "Absorbing boundary conditions for elastic waves", Geophysics., Vol. 56, pp. 231-41, 1991.

[30] C. Cerjan, D. Kosloff, R. Kosloff, and M. Reshef, "A non-reflecting boundary condition for 
discrete acoustic and elastic wave equation", Geophysics., Vol. 50, pp. 705-8, 1985.

[31] J. P. Bérenger, "A perfectly match layer for the absorption of electromagnetic waves", $J$. of computational physics., pp. 185-200, 1993.

[32] D. Komatitsch and R. Martin, "An unsplit convolutional perfectly matched layer improved at grazing incidence for seismic wave equation", Geophysics., Vol. 72, issue 5, pp. SM155SM167, 2007.

[33] Y. Liu and M. K. Sen, "Advanced finitedifference methods for seismic modelling", Geohorizons, 2009.

[34] W. C. Chew and Q. H. Liu, "Perfectly Matched layer for elastodynamics: a new absorbing boundary condition", J. Comp. Acoust., 1996.

[35] Y. Liu and M. K. Sen., "A hybrid absorbing boundary condition for elastic wave modelling with staggered-grid finite difference", In: Proc. of SEG Denver annual meeting, Denver, Colorado, USA, pp.2945-2949, 2010.

[36] J. Kristek, P. Moczo, and M. Galis, "A Brief summary of some PML formulations and discretizations for the velocity-stress equation of seismic motion", Stud. Geophys. Vol. 53, No.6, pp. 459-474, 2009.

[37] Q. H. Lui and J. Tao, "The perfectly matched layer for acoustic waves in absorptive media", $J$. Acoust. Soc. Am. Vol. 102, No. 4, pp. 2072-2082, 1997.

[38] B. Hustedt, S. Operto, and J. Virieux, "Mixedgrid and staggered grid finite-difference methods for frequency-domain acoustic wave modelling", J. Geophys. Int, 157, pp. 1269-1296. doi:10.1111/j.1365-246X.2004.02289.x, 2004.

[39] Y. Gao, H. Song, J. Zhang, and Z. Yao, "Comparison of artificial absorbing boundaries for acoustic wave equation modeling", CSIRO J. Exp. Geophys., http://dx.doi.org/10.1071/EG15068, 2018.

[40] F. Collino and C. Tsogka, "Application of perfectly matched absorbing layer model to the linear electrodynamic problem in anisotropic heterogeneous media", J., pp. 1-16, 2000. 